Itinéraires Itinéraires

Littérature, textes, cultures

\title{
La volonté balzacienne comme donnée formelle de La Comédie humaine
}

\section{Yannick Roy}

\section{OpenEdition}

\section{Journals}

Édition électronique

URL : http://journals.openedition.org/itineraires/2147

DOI : $10.4000 /$ itineraires. 2147

ISSN : 2427-920X

Éditeur

Pléiade

\section{Édition imprimée}

Date de publication : 1 mai 2010

Pagination : $31-39$

ISBN : 978-2-296-11224-7

ISSN : 2100-1340

\section{Référence électronique}

Yannick Roy, "La volonté balzacienne comme donnée formelle de La Comédie humaine », Itinéraires [En ligne], 2010-1 | 2010, mis en ligne le 01 mai 2010, consulté le 23 avril 2019. URL : http://

journals.openedition.org/itineraires/2147; DOI : 10.4000/itineraires.2147

\section{(ब) $\Theta \Theta$}

Itinéraires est mis à disposition selon les termes de la licence Creative Commons Attribution - Pas d'Utilisation Commerciale - Pas de Modification 4.0 International. 


\title{
La volonté balzacienne comme donnée formelle de La Comédie humaine
}

\begin{abstract}
Balzac was not an observer as much as a visionary or a seer, who invented his characters not by basing them on external models, but by becoming them to the point of losing his own identity. That may at first seem to question the credibility of his fiction, but what is left of the author in each of his characters is a quality so tenuous that it does not allow us to recognize him. This quality can be said to be his will, understood here not as a subject matter but as an element of the form, resembling the sort of availability in which Jacques Rivière saw the essence of the adventure novel. Balzac's method thus forces him to relinquish all attempts at embracing the totality of his Human Comedy, which he builds from the inside without ever seeking to control it.
\end{abstract}

Keywords : suspension of disbelief, fiction, creation, imaginary world, Balzac Mots clés : suspension de l'incrédulité, fiction, création, monde imaginaire, Balzac

Il est difficile de se représenter Balzac au travail sans se heurter à un paradoxe dont les termes ont été admirablement fixés par Baudelaire, dans un passage bien connu de l'essai qu'il a consacré à Théophile Gautier ${ }^{1}$ :

J'ai maintes fois été étonné que la grande gloire de Balzac fût de passer pour un observateur; il m'avait toujours semblé que son principal mérite était d'être visionnaire, et visionnaire passionné. Tous ses personnages sont doués de l'ardeur vitale dont il était animé lui-même. Toutes ses fictions sont aussi profondément colorées que les rêves. Depuis le sommet de l'aristocratie jusqu'aux bas-fonds de la plèbe, tous les acteurs de sa Comédie sont plus âpres à la vie, plus actifs et rusés dans la lutte, plus patients dans le malheur,

1. Albert Béguin a placé ce passage en exergue de son Balzac lu et relu, Paris, Seuil, coll. « Pierres Vives », 1965. 
plus goulus dans la jouissance, plus angéliques dans le dévouement, que la comédie du vrai monde ne nous les montre. Bref, chacun, chez Balzac, même les portières, a du génie. Toutes les âmes sont des armes chargées de volonté jusqu'à la gueule. C'est bien Balzac lui-même².

On comprend que l'auteur des Fleurs du Mal tranche la question ainsi et accorde à Balzac la distinction d'être un visionnaire, qualité plus rare, et sans doute aux yeux de Baudelaire plus méritoire, que celle d'observateur. On peut difficilement ne pas lui donner raison : dès lors qu'on s'interroge sur le comment de La Comédie humaine, dès lors qu'on s'intéresse à la manière dont Balzac, concrètement, la plume à la main pour ainsi dire, a fabriqué son œuvre, 1'hypothèse d'un historien patient qui recueille laborieusement des observations sur sa société afin d'en brosser le tableau paraît indéfendable, voire extravagante - alors qu'elle demeure valable, partiellement du moins, chez Flaubert par exemple, ou encore chez Zola. Il est vrai que Balzac s'est lui-même représenté, dans son célèbre « Avant-propos », sous les traits d'un historien, ou d'un simple secrétaire écrivant sous la dictée de la Société $^{3}$; mais les seules dimensions de son œuvre, sans parler de sa teneur, de son contenu, de son désordre foisonnant, et surtout peut-être de cette qualité subtilement onirique dont parle Baudelaire, nous disent autre chose et laissent croire à quelque jaillissement plus inspiré ou plus spontané.

Baudelaire n'est d'ailleurs pas le seul à l'avoir noté ; on peut également citer à cet égard le témoignage de Théophile Gautier, qui lui aussi dépeint Balzac comme un visionnaire, plus précisément comme un voyant, et lui prête un « don d'avatar », c'est-à-dire la capacité « de s'incarner dans des corps différents et d'y vivre le temps qu'il voulait » :

Son mérite d'observateur, sa perspicacité de physiologiste, son génie d'écrivain ne suffisent pas pour expliquer l'infinie variété des deux ou trois mille types qui jouent un rôle plus ou moins important dans la Comédie humaine. Il ne les copiait pas, il les vivait idéalement, revêtait leurs habits, contractait leurs habitudes, s'entourait de leur milieu, était eux-mêmes tout le temps nécessaire ${ }^{4}$.

Bien qu'il insiste un peu plus sur ce qui distingue les personnages balzaciens les uns des autres, et bien qu'il reconnaisse à l'auteur de La Comédie humaine un certain mérite d'observateur, Gautier dit essentiellement la même chose que Baudelaire, à savoir que Balzac vivait à la place de ses personnages, se

2. Baudelaire, "Théophile Gautier», dans Euvres complètes, Paris, Robert Laffont, coll. « Bouquins », 1980, p. 502.

3. Balzac, «Avant-propos », dans La Comédie humaine, t. I : Études de mours : scènes de la vie privée, éd. Pierre-Georges Castex, Paris, Gallimard, coll. "Bibliothèque de la Pléiade », 1976, p. 11.

4. Théophile Gautier, Balzac [1860], éd. Jean-Luc Steinmetz, Paris, Le Castor Astral, coll. « Les inattendus », 1999, p. 35-36. L'auteur souligne. 
confondait avec eux, leur prêtait sa volonté ou sa sensibilité, et que c'était là en quelque sorte sa méthode de travail.

Bien sûr l'admiration de Gautier et de Baudelaire pour Balzac est aussi grande que sincère, et ni l'un ni l'autre ne songe à lui reprocher d'être un visionnaire ou un voyant, mots qui sont presque synonymes ici de " génie ». Pourtant cet éloge est peut-être un peu ambigu, et peut même, dès lors qu'il est adressé à un romancier plutôt qu'à un poète, être interprété comme un subtil constat d'échec. "C'est bien Balzac lui-même ", dit Baudelaire en parlant indistinctement de tous les personnages de La Comédie humaine; et Gautier, évoquant lui aussi tous ces personnages et cherchant à définir le lien qui unit Balzac à cette foule, trouve cette phrase curieuse, presque boiteuse : « il [...] était eux-mêmes ». Ce passage intempestif du singulier au pluriel résume le problème; penser à La Comédie humaine, c'est sans doute penser à une multitude de personnages, à une société entière dont la totalité se dérobe sous les drames innombrables qu'elle abrite et qui mobilisent sans cesse l'attention du lecteur; mais c'est aussi, surtout peut-être, quand cette attention se relâche quelque peu et favorise un certain recul, entrevoir derrière cette pluralité foisonnante un seul esprit, un seul style, une seule personnalité, celle de Balzac, toujours la même sous ses incarnations diverses. On voit à quelle conclusion ceci peut conduire; s'il est vrai qu'un romancier demande à ses lecteurs de « croire » aux histoires qu'il leur raconte et de faire comme si les personnages à qui elles arrivent existent réellement, que penser d'un romancier qui soit à ce point reconnaissable derrière les masques de ses créatures? Débusquer le génie de Balzac derrière ces dandys, ces usuriers, ces femmes perdues, ces journalistes, ces actrices, ces figures angéliques, ces travailleurs acharnés, ces grands criminels et ces petits escrocs, n'estce pas dire que La Comédie humaine est cousue de fil blanc, qu'elle est au mieux la rêverie d'un seul homme, au pire une immense supercherie, et que s'il est permis de s'y intéresser il ne saurait précisément être question d'y « croire »? On se trouve ici, en somme, devant une alternative : ou bien on plonge dans la multitude, et dès lors on oublie un peu Balzac en faveur de sa foisonnante Comédie, ou bien on reste en retrait, sur la touche en quelque sorte, à considérer l'étonnant rêveur qui a imaginé tout cela - à le considérer avec admiration, certes, mais avec une admiration qui, insensiblement, tend à faire de lui un personnage (à vrai dire le seul personnage de l'œuvre, celui qui est simultanément tous les autres) plutôt qu'un romancier.

La question, il va sans dire, ne doit pas être confondue avec celle de la vraisemblance, et à ce titre même la notion d'observation est ici secondaire. Il ne s'agit pas de savoir si ce qui arrive dans le monde de La Comédie humaine aurait pu arriver dans le monde réel, ni d'évaluer les mérites de Balzac en tant qu'observateur ou historien des mœurs de son temps, mais de mesurer la crédibilité de la fiction balzacienne, l'adhésion plus ou moins grande qu'elle suscite chez le lecteur, ou le type d'attention qu'elle exige de lui. Baudelaire et Gautier semblent éluder la question. Tous deux éclairent 
la fabrication de l'œuvre et attirent l'attention sur ce qui se passe dans les coulisses de La Comédie humaine, c'est-à-dire dans la conscience créatrice de Balzac, mais sans s'attarder aux conséquences d'une telle attention sur le lecteur qui, victime consentante de l'illusion romanesque, aperçoit les personnages au milieu d'un décor auquel il croit, sur une scène dont l'éclat rejette provisoirement la réalité dans l'ombre. On s'étonne même de constater que Gautier souligne, tout de suite après avoir décrit la méthode de Balzac et comme s'il n'y avait là rien de contradictoire, à quel point les personnages de La Comédie humaine lui paraissent vivants : «Un véritable sang rouge circule dans leurs veines au lieu de l'encre qu'infusent à leurs créations les auteurs ordinaires ${ }^{5}$. $\gg$ Certes, la remarque n'est pas incongrue si l'on s'en tient à l'argument implicite voulant que ces personnages, imaginés de l'intérieur, « vécus idéalement », paraissent naturellement plus vivants que s'ils avaient été décrits de l'extérieur et animés laborieusement comme des automates. Mais peut-être faut-il alors distinguer cette vie apparente de la crédibilité dont il vient d'être question; est-ce bien croire à leur existence que de les trouver vivants quand on sait que cette vie est celle de leur créateur, et que le « véritable sang rouge » circulant dans leurs veines est celui de Balzac?

Le silence de Gautier et de Baudelaire n'est pourtant pas inexplicable; le fait qu'ils ne relèvent pas la contradiction ne fait que souligner négativement, en quelque sorte, la banalité de ce qui se passe concrètement dans l'esprit d'un lecteur de roman. L'expérience qui consiste à imaginer des personnages et à leur prêter vie est une des plus courantes qui soient; elle est à vrai dire si courante qu' on a du mal à concevoir une conscience incapable de l'abandon minimal qu'elle exige, c'est-à-dire entièrement dépourvue d'imagination. Cette activité s'apparente à la rêverie, se confond presque avec la distraction, et s'accommode fort bien des contradictions qui semblent la rendre impossible aux yeux d'un analyste trop pointilleux ou trop catégorique. L'attitude tranchante adoptée par René Girard dans Mensonge romantique et vérité romanesque nous semble à cet égard exemplaire; soulevant une question semblable à la nôtre, quoique d'un point de vue plus général, Girard refuse d'admettre simultanément la liberté de l'auteur et celle des personnages :

L'influence existentialiste a mis à la mode le mot de « liberté ». On nous dit tous les jours que le romancier ne parvient au génie qu'en « respectant» la liberté de ses personnages. On ne nous dit jamais, malheureusement, en quoi peut bien consister ce respect. La notion de liberté est forcément ambiguë quand on l'applique au roman. Si le romancier est libre on voit mal comment ses personnages le seraient. La liberté ne se partage pas, même entre créature et créateur. C'est là un dogme fondamental et M. Jean-Paul Sartre se fait fort, grâce à lui, de prouver l'impossibilité d'un Dieu créateur. Ce qui est impossible à Dieu ne saurait être possible au romancier. Ou bien le 
romancier est libre et ses personnages ne le sont pas, ou bien les personnages sont libres et le romancier, tout comme Dieu, n'existe pas ${ }^{6}$.

La conclusion de Girard est sans appel, et on voit mal comment on pourrait réfuter un raisonnement aussi simple et aussi péremptoire. Mais si la valeur intrinsèque du raisonnement n'est pas en cause, sa pertinence ne va pas de soi. La logique, bien entendu, voudrait qu' on choisisse entre l'une ou l'autre de deux propositions contradictoires; mais un lecteur de roman n'est pas tenu de se plier à la logique et de purger son esprit de toute contradiction. La crédibilité d'une fiction n'exige pas l'oubli de tout ce qui existe en dehors d'elle, ou disons de tout ce qui devrait logiquement inciter le lecteur à cesser d'y croire; elle suppose plutôt l'attitude à laquelle Coleridge donnait le nom de « foi poétique », et qu'il décrivait comme une « suspension temporaire de l'incrédulité ${ }^{7} »$. La lecture d'un roman n'est pas incompatible avec une certaine conscience des moyens mis en œuvre par le romancier pour faire illusion. D'ailleurs il tombe sous le sens que cette activité s'effectue toujours dans la durée, et que par conséquent l'adhésion du lecteur peut varier en intensité. Entièrement absorbé par la fiction, rien ne l'empêche, une fois le livre fermé, de réfléchir au travail du romancier; cette réflexion ne risque guère de compromettre la crédibilité des personnages, ni le plaisir qu'il aura à se replonger dans leur histoire.

Mais il y a, dans le cas de Balzac, une autre raison, plus subtile mais plus fondamentale, de ne pas opposer aussi catégoriquement le lecteur naïf au lecteur lucide, c'est-à-dire celui qui « croit » aux personnages à celui qui ne «croit» qu'au romancier. Le lien de parenté qui unit Balzac à ses personnages est en effet bien ténu, et ce qui « reste » du créateur dans chacune de ses créatures bien difficile à définir. Nous avons parlé plus haut de la personnalité de Balzac, et affirmé que cette personnalité était reconnaissable derrière chacun de ses personnages; mais ce sont là des mots qui en disent sans doute un peu plus qu'il ne faudrait. Que représente exactement la métaphore du " véritable sang rouge » dont parle Gautier, et que le romancier visionnaire partage avec ses incarnations fictives? De toute évidence il ne saurait s'agir de quelque donnée biographique ni de quelque trait physique que ce soit, et même les mots « caractère », « personnalité », « tempérament » paraissent désigner des ensembles de qualités accessoires, superficielles et contingentes en regard de celle que Balzac prête à ses héros. Baudelaire en donne sans doute l'idée la plus juste en parlant d' " ardeur vitale », d' " âpreté »,

6. René Girard, Mensonge romantique et vérité romanesque, Paris, Grasset, coll. « Pluriel », 1961, p. 289.

7. Samuel Taylor Coleridge, Biographia literaria [1907], vol. II, éd. J. Shawcross avec Aesthetical Essays, London, Oxford University Press, 1969, p. $6:$ : ... my endeavours should be directed to persons and characters supernatural, or at least romantic; yet so as to transfer from our inward nature a human interest and a semblance of truth sufficient to procure for these shadows of imagination that willing suspension of disbelief for the moment, which constitutes pœtic faith. » 
de « patience », de « dévouement », et en résumant tous ces termes par ceux de " génie » et de « volonté ». Il est frappant de constater avec lui qu'on ne trouve dans La Comédie humaine aucun personnage authentiquement velléitaire, ou disons, pour préserver l'indétermination qu'exige notre propos, aucun personnage dépourvu d'énergie, d'intensité, de vitalité; l'extrémité même de la mélancolie ne semble jamais intéresser Balzac qu'au moment où le héros tente de se résoudre au suicide et s'apprête par là à faire preuve de volonté une dernière fois, comme Lucien de Rubempré à la prison de la Conciergerie. Mais cette qualité ineffable - qu'on lui donne le nom de volonté, de génie ou d'ardeur - ne suffit pas à définir un personnage. En ce sens la « ressemblance » perçue par Baudelaire, s'il est permis d'employer ce mot, est pour ainsi dire d'une généralité insurmontable : elle est évidente - du moins quand on s'en est avisé une première fois - mais en même temps elle est invisible tant elle touche à une qualité virtuelle, qui ne détermine presque rien et peut s'actualiser de mille et une façons, donner lieu à des caractères infiniment divers et engendrer des personnages qui, si l'on veut bien nous permettre cette tournure paradoxale, se ressemblent tous sans avoir l'air de se ressembler.

Le plus étonnant, d'ailleurs, est que Balzac lui-même ne s'est jamais véritablement reconnu en eux. Mieux placé que quiconque, à l'évidence, pour connaître le fin mot de l'histoire et pour savoir ce qu'il en est de la « supercherie » dont nous parlons ici, il semble plutôt en avoir été la première dupe. On ne peut omettre d'évoquer, sur la question de sa « méthode », la description fameuse - d'ailleurs citée et commentée par Gautier - sur laquelle s'ouvre Facino Cane, et qui donne de la nature véritable de son travail une idée plus précise et plus concrète que le texte programmatique et quelque peu artificiel de l' « Avant-propos ». Comme Gautier et Baudelaire, Balzac se décrit comme un visionnaire ou un voyant; s'il se propose explicitement, dans ces pages remarquables, d'observer les mœurs de son temps, c'est par des moyens quelque peu surnaturels, c'est-à-dire grâce à ce qu'il appelle sa « seconde vue » :

Une seule passion m'entraînait en dehors de mes habitudes studieuses; mais n'était-ce pas encore de l'étude? J'allais observer les mœurs du faubourg, ses habitants et leurs caractères. [...] Chez moi, l'observation était déjà devenue intuitive, elle pénétrait l'âme sans négliger le corps; ou plutôt elle saisissait si bien les détails extérieurs, qu'elle allait sur-le-champ audelà; elle me donnait la faculté de vivre de la vie de l'individu sur laquelle elle s'exerçait, en me permettant de me substituer à lui comme le derviche des Mille et une Nuits prenait le corps et l'âme des personnes sur lesquelles il prononçait certaines paroles ${ }^{8}$.

8. Balzac, Facino Cane, dans La Comédie humaine, t. VI : Études de mœurs : scènes de la vie parisienne (suite), op. cit., p.1019-1020. 
Force est d'admettre - à moins de croire aux phénomènes surnaturels - que ce que Balzac appelle ici « étude » ou « observation » est en réalité une forme de rêverie beaucoup plus libre et beaucoup plus solitaire, qui n'a pu être élaborée qu'à l'écart de la réalité; mais cette distinction ne semble pas avoir cours dans l'esprit de Balzac, parfaitement convaincu non pas d'inventer la vie d'inconnus croisés par hasard et autour desquels se cristalliseraient les produits de son imagination (ce qui serait déjà remarquable), mais bien de traverser les apparences et d'observer ces inconnus tels qu'ils sont (ce qui tient du prodige). La notion même de "seconde vue », l'assimilation de cette forme de rêverie à une méthode d'observation, et surtout la manière dont il en parle, c'est-à-dire avec l'assurance de celui qui, effectivement, voit, sent et perçoit la réalité, semblent indiquer qu'il a bel et bien cru à la validité objective des données que cette méthode lui permettait de recueillir. Les créatures qu'il « investit » subjectivement ne sont pas à ses yeux des créatures imaginaires, mais des créatures bien réelles qui, aussitôt inventées, c'est-à-dire habitées par Balzac, se détachent de lui pour mener une existence objective, comme ce vieux clarinettiste aperçu lors d'une noce et devenu, par la magie de la " seconde vue », Facino Cane, principe de Varese. L'observation et l'introspection ne sont pour Balzac qu'une seule et même chose ; il est permis d'y voir une certaine naïveté, mais sans cette naïveté La Comédie humaine aurait été inconcevable. Ayant lui-même, de son propre aveu, « vécu » d'une autre vie que la sienne, il l'oublie aussitôt et découvre cette vie en face de lui, dans toute son irréductible altérité. On ne compte pas les anecdotes qui témoignent du mélange constant qui s'opérait chez lui entre la fiction et la réalité, la plus célèbre étant sans doute celle, probablement apocryphe mais reprise par tous ses biographes, voulant qu'il ait, à l'article de la mort, fait demander à son chevet Horace Bianchon ${ }^{9}$, celui-là même qui a diagnostiqué le mal de Louis Lambert et accompagné le Père Goriot dans son agonie.

Cette espèce de « naïveté géniale » nous ramène à notre problème, qui se pose pour ainsi dire avec une acuité accrue : si Balzac lui-même évite de se confondre avec les personnages auxquels il s'est pourtant « substitué », s'il est en mesure de s'abandonner provisoirement à une forme de donquichottisme sur lequel il ne cesse d'exercer un mystérieux contrôle, si même après avoir prêté sa vie à ses créatures il ne perd jamais de vue sa propre identité et continue de voir ses personnages comme des individus

9. L'origine probable de cette anecdote, reprise entre autres par René Benjamin (La vie prodigieuse de Balzac, Paris, Plon, 1925 et coll. «10/18», 1962, p. 310), Stefan Zweig (Balzac, le roman de sa vie, Paris, Albin Michel, 1950 et Le livre de poche, 1996, p. 491) et André Maurois (Prométhée ou la vie de Balzac, Hachette, 1965, p. 595) se trouve vraisemblablement dans le récit de la mort de Balzac inséré par Octave Mirbeau dans son roman intitulé $L a$ 628-E8. Les chapitres de ce roman qui concernent Balzac ont été publiés à part : Octave Mirbeau, La Mort de Balzac, suivi de Une publication scandaleuse, par P. Michel et J.-F. Nivet, Paris, Éditions du Félin et Arte Éditions, 1999. 
distincts qui existent objectivement, c'est que cette vie qu'il leur a prêtée, justement, n'est pas à proprement parler la sienne. Il ne s'agit pas d'une vie au sens « biographique » du terme - d'une vie faite d'événements, de traits de caractères et de données objectives - mais bien de la vie en général, ou si l'on veut d'une espèce de souffle, de fluide vital, anonyme et universel, qui ne contribue en rien à singulariser l'individu ni à fonder son identité. Cette énergie n'est pas assimilable à quelque donnée thématique ni à quelque contenu que ce soit; en ce sens on peut dire qu'elle ne relève pas du fond de l'œuvre, ni de son sujet, ni de sa matière, mais de sa forme même.

Jacques Rivière a publié, en 1913, un célèbre essai sur le roman d'aventure dont le propos nous semble coïncider en partie avec les considérations qui précèdent sur la création balzacienne. On sait que l'expression « roman d'aventure » avait pour Rivière une portée plus vaste que celle que lui reconnaît l'usage courant; il ne s'agissait pas seulement, disons, de romans d'aventure au sens strict, comme ceux de Stevenson, Kipling, Dumas ou Verne, mais d'un certain idéal esthétique dont la validité semble s'étendre, bien souvent, au roman en général ${ }^{10}$. Cet idéal découle d'un certain bonheur de la découverte et de l'improvisation; le roman d'aventure "s'avance à coups de nouveauté ${ }^{11} »$, et à ce titre marque une rupture décisive par rapport à la sensibilité délétère de l'époque symboliste, qui, dans la perspective de Rivière, s'achève au moment où il écrit son essai. À la conscience excessive des poètes symbolistes, qui épuisèrent le réel ou le rendirent inintéressant à force d'intelligence, Rivière oppose $1^{\prime}$ ' « ignorance et la naïveté ${ }^{12}$ » de son romancier idéal, à qui il va jusqu'à prêter « une sorte de stupidité qui fait corps avec son pouvoir créateur ${ }^{13} »$. Ce romancier ne domine jamais son œuvre, demeure « tout confondu » avec elle, «ne la déborde d'aucun côté ${ }^{14} »$, et s'y abandonne sans savoir où elle le mènera. C'est dans cette

10. À vrai dire cette extension est un peu problématique, dans la mesure où Rivière parle d'une littérature qui n'existe pas encore, qu'il appelle de ses vœux, ou plutôt dont il annonce l'avènement imminent, mais qu'il décrit en ne quittant pour ainsi dire jamais le terrain des généralités abstraites. Les exemples qu'il donne à l'appui de son propos étant peu nombreux et sommairement esquissés, il est difficile de lire ce texte brillant sans être tenté d'y « insérer » ses propres exemples, c'est-à-dire sans appliquer les idées et les observations de Rivière à des œuvres auxquelles, vraisemblablement, il ne songeait pas, comme justement La Comédie humaine. On se sent d'autant plus autorisé à réaliser une telle substitution que le texte de Rivière porte peut-être moins sur une catégorie donnée d'œuvres littéraires que sur un certain espoir, ou une certaine attente à l'égard des romans qui s'écrivaient alors, ou qui auraient dû s'écrire, et qu'en tout cas il aurait souhaité lire; autrement dit Rivière décrit moins un genre qu'une certaine disposition d'esprit, toute subjective, qui est bien sûr celle des nouveaux écrivains, mais aussi celle des nouveaux lecteurs.

11. Jacques Rivière, Le Roman d'aventure, postface d'Alain Clerval, Paris, Éditions des Syrtes, 2000, p. 68.

12. Ibid. p. 66.

13. Ibid. p. 56.

14. Ibid. p. 57. 
disponibilité aveugle que se trouve l'essence du roman d'aventure. La notion même ne se rattache pas à telle ou telle constellation de thèmes plus ou moins originaux, mais à une sorte de sinuosité ou d'imprévisibilité; c'est pourquoi Rivière peut dire de l'aventure ce que nous avons dit de l'énergie balzacienne, à savoir qu'elle « est la forme de l'œuvre plutôt que sa matière ${ }^{15} \gg$. Les deux principes ont ceci en commun qu'ils impliquent de la part de l'artiste une certaine immersion dans l'œuvre, et par là même un certain renoncement à sa totalité.

L'idée d'un tel renoncement est à première vue difficilement applicable à Balzac, qui a souvent affiché ses ambitions totalisantes et déclaré plus d'une fois son intention de faire de La Comédie humaine un monde à part entière, posé à côté du monde réel et pouvant être saisi comme tel, dans sa globalité. Mais il est sans doute permis, et même souhaitable, de distinguer ce que Balzac a fait de ce qu'il disait vouloir faire. S'il a pu rêver, suivant la formule célèbre qui se trouve dans une lettre à Madame Hanska datée de 1844 , de porter « une société tout entière dans sa tête... ${ }^{16}$ », il est certain qu'il n'a jamais réalisé ce rêve dans toute sa plénitude. D'abord bien sûr parce qu'une « société tout entière » est un objet complexe et divisible à l'infini, qui ne saurait tenir dans une tête, fût-elle celle de Balzac ; mais aussi et surtout parce que ce désir même de contenir ou de dominer La Comédie humaine, s'il s'exprime parfois dans des lettres ou dans des textes liminaires, comme celui de 1' " Avant-propos », est incompatible avec sa méthode, et par conséquent remarquablement peu visible dans ses romans. Là se trouve au fond la clé du paradoxe dont il a été question plus haut; Balzac s'égare ou se dissémine dans une œuvre qu'il construit de l'intérieur mais qu'il ne parvient jamais à maîtriser. En se confondant subjectivement avec ses personnages, il se rend peut-être visible, à tout le moins pour des lecteurs perspicaces comme Baudelaire et Gautier; mais il ne se rend visible qu'au moment de s'enraciner profondément dans la fiction sous l'aspect de tel ou tel personnage, et par le fait même ne cesse de se dérober comme romancier. Si La Comédie humaine est comparable à un monde, ne serait-ce que par ses dimensions, il importe peut-être d'ajouter que ce monde n'est véritablement perceptible que dans la proximité, de manière fragmentaire - dans les détails, les vies, les destins, les histoires qui en constituent la trame - et jamais dans sa totalité de monde; d'ailleurs il ressemble ainsi d'autant plus au monde réel, qui lui non plus ne se laisse pas embrasser d'un seul regard.

Yannick Roy

Revue L'Inconvénient, Montréal

15. Ibid.p. 69.

16. Balzac, Lettres à Madame Hanska, Paris, Robert Laffont, 1990, coll. « Bouquins », p. 804. 